\title{
Effects of Water Pressure during Polymerization on Mechanical Properties of Autopolymerized Resins
}

\author{
Yasuo Toyoda, DDS, PhD, ${ }^{a}$ Makoto Oizumi, DDS, PhD, ${ }^{\text {a }}$ Tetsuya Suzuki, DDS, PhD, ${ }^{a}$ \\ Hidekazu Takahashi, DDS, PhD, ${ }^{b}$ Nobusuke Oda, DDS, PhD, ${ }^{a}$ Takuya Ohkubo, DDS, ${ }^{a}$ \\ and Junichi Furuya, DDS, $\mathrm{PhD}^{\mathrm{a}}$ \\ ${ }^{a}$ Department of Removable Prosthodontics, School of Dentistry, Iwate Medical University, Morioka, Japan \\ ${ }^{\mathrm{b}}$ Advanced Biomaterials, Department of Restorative Science, Division of Oral Health Science, Graduate \\ School, Tokyo Medical and Dental University, Tokyo, Japan
}

\section{Clinical significance}

This study suggests that application of water pressure during polymerization improves the mechanical properties of autopolymerized resins, offering benefits for long-term usage, better aesthetics of provisional restorations, and repairing dentures.

\begin{abstract}
Purpose: This study evaluated the effects of water pressure on the mechanical properties and tensile bond strength of autopolymerized resins during polymerization at room temperature using a dental pressure pot with chair-side ease of handling.

Methods: Four different autopolymerized resins (Unifast II, Unifast III, Provinice, and Miky Plus) were used. Specimens were fabricated by the brushon technique. In the pressure group, specimens were polymerized in a dental pressure pot under $0.2 \mathrm{MPa}$ water pressure at room temperature. In the non-pressure group, specimens were polymerized on bench. The 2 groups were compared for dimensional accuracy, 3-point bending, and tensile bond strength. Data were statistically analyzed by two-way analysis of variance (ANOVA) with Tukey's multiple comparison test at a significance level of $\alpha=0.05$.

Results: Two-way ANOVA indicated that both resin and pressure had significant effects on the polymerization shrinkage rate (PSR). Interaction between resin and pressure was also significant. The PSR was lower in the pressure group than in the non-pressure group. The PSRs of Unifast II and Miky Plus were greatly improved by pressure polymerization. Significant differences in flexural strength were observed between the 2 groups. The flexural strength significantly in-
\end{abstract}

\section{Corresponding to: Dr Yasuo Toyoda}

Department of Removable Prosthodontics, School of

Dentistry, Iwate Medical University

1-19 Uchimaru, Morioka 020-8505, Japan

Tel: +81-19-651-5111, Fax: +81-19-652-3820

E-mail: ytoyoda@iwate-med.ac.jp

Received on March 22, 2007 / Accepted on June 8, 2007 creased under pressure. No significant differences in tensile bond strength were observed between the 2 groups.

Conclusion: The dimensional accuracy and flexural strength of autopolymerized resins were significantly improved by the application of water pressure during polymerization. No significant changes in tensile bond strength were observed following pressure application at room temperature.

Key words: autopolymerized resin, flexural strength, tensile bond strength, dimensional accuracy, water pressure

\section{Introduction}

Autopolymerized resins have numerous chairside applications, including fabricating provisional restorations and repairing dentures. Provisional restorations require good esthetic quality, wear resistance, and fracture toughness, particularly in the case of long-span bridges. In order to ensure the longevity of denture repairs, autopolymerized resins should possess superior mechanical properties and greater bonding strength to denture base resins. However, due to their relatively large residual monomer content and discoloration by terinary amines, autopolymerized resins originally have lower strength, durability, and aesthetic quality than heat-polymerizing resins and light-polymerizing resins. Furthermore, autopolymerized resins tend to become contaminated by air bubbles. Meanwhile, autopolymerized resins also have some advantages with regard to rapid chair-side management because of rapid polymerization, ease of handling, and inexpensive cost.

There is a possibility of improvement of the low mechanical properties of resins by applica- 
tion of heat or pressure during polymerization in a hydroflask. ${ }^{1-3}$ If autopolymerized resins can be polymerized by the application of pressure in clinical settings easily, the process will effectively reduce porosity, ${ }^{4,5}$ resulting in a smooth polymerized resin surface. It is even possible that the region of denture repair will be indistinguishable from the normal dentition, thereby improving aesthetic appearance.

Some reports have examined the influence of curing environments such as water temperature, pressure curing, and repair surface design on the flexural strength and bond strength of the repaired region. ${ }^{6-10}$ However, the changes in the mechanical properties of autopolymerized resins by application of water pressure during polymerization have not been sufficiently elucidated.

A dental pressure pot with chair-side ease of handling has been introduced recently. In the present study, using the dental pressure pot during polymerization, we focused on the effects of water pressure alone at room temperature on the mechanical properties and tensile bond strength of autopolymerized resins.

\section{Materials and methods}

\section{Autopolymerized resins and polymerization methods}

Four different autopolymerized resins (Unifast II, Unifast III, Provinice, and Miky Plus; Table 1 were used in the experiments. All specimens used in the tests were fabricated by the brush-on technique; half the specimens were polymerized under pressure (pressure group), and the remaining were polymerized without applying pressure (non-pressure group). In the pressure group, the specimens were polymerized using a dental pressure pot (New Pressure Pot; Bosworth, Skokie, Illinois) under $0.2 \mathrm{MPa}$ water pressure at room temperature $\left(23^{\circ} \mathrm{C}\right)$. This pressure pot has chairside ease of handling since it does not require heat, electricity, and compressed air. In the nonpressure group, the specimens were polymerized on bench at room temperature. The 2 groups were compared using the dimensional accuracy test, 3-point bending test, and tensile bond test. Six specimens made of each autopolymerized resin were examined in each test.

In a preliminary experiment, the setting times of all resins were measured with a thermocouple sensor. The curing time was defined as the $\mathrm{du}^{-}$
Table 1 Autopolymerized resins used.

\begin{tabular}{llc}
\hline Trade name & \multicolumn{1}{c}{ Manufacturer } & Lot No. (Powder/Liquid) \\
\hline Unifast II & GC, Tokyo, Japan & $508301 / 606231$ \\
Unifast III & GC, Tokyo, Japan & $606151 / 605302$ \\
Provinice & Shofu, Kyoto, Japan & $90403 / 60698$ \\
Miky Plus & Nissin, Kyoto, Japan & GFA/GIF \\
\hline
\end{tabular}

ration of time from the start of brushing on the teflon tube to the time of recording the highest temperature due to exothermic chemical reaction. Five specimens made of each resin were assessed. There were significant differences among the 4 resins. The means and standard deviations of the setting times of Unifast III, Unifast II, Provinice, and Miky were 164.3 $\pm 17.7,200.9 \pm 10.4$, $258.4 \pm 6.8$, and $321.7 \pm 22.4$ seconds, respectively. Based on these results, the time to place the specimens into the pressure pot was determined based on Unifast III that showed the shortest setting time. The surface of the Unifast III resin mixture prepared by the brush-on technique was examined with a scanning electron microscope. The resin mixtures were placed in the dental pressure pot for 5 minutes duration at 6 different time points: at 15 seconds, 30 seconds, 1 minute, 2 minutes, 3 minutes, and 5 minutes of starting the procedure. The shapes of the polymer particles on the surface were notably different at 30 seconds and at 1 minute after starting the procedure (Fig. 1). Based on this finding, all specimens in the present study were placed into the pressure pot within 30 seconds after brushing on.

\section{Dimensional accuracy test}

Figure 2 shows the schematic illustration of a trapezoidal stainless-steel mold (base length: 25 mm; upper rim: $24 \mathrm{~mm}$; height: $10 \mathrm{~mm}$ ) and a specimen. The mold was placed on a glass slide, and autopolymerized resin was filled into the mold using the brush-on technique. In the pressure group, the mold was placed into the dental pressure pot within 30 seconds of starting the filling. In the non-pressure group, the mold was left on bench. Ten minutes after completion of resin filling, the polymerized resin specimen was removed from the mold and immersed in purified water at $37^{\circ} \mathrm{C}$ for 24 hours. The specimens were placed into the stainless steel mold, and the gaps between the bottom edge of the mold and the specimens were measured with a measuring microscope (MM60; Nikon, Tokyo, Japan) at 3 


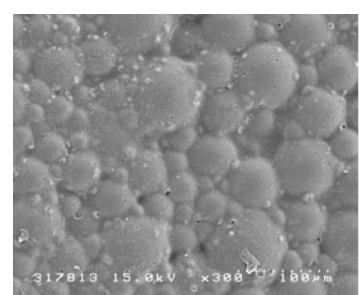

$15 \mathrm{~s}$

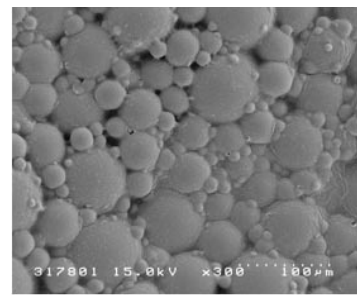

$2 \min$

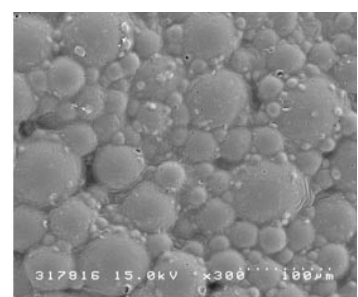

$30 \mathrm{~s}$

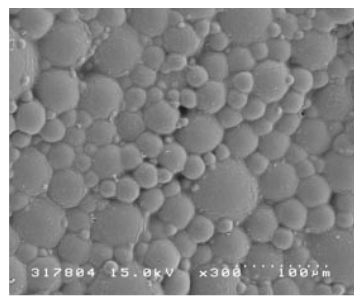

$3 \mathrm{~min}$

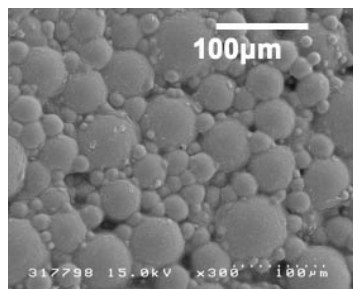

$1 \mathrm{~min}$

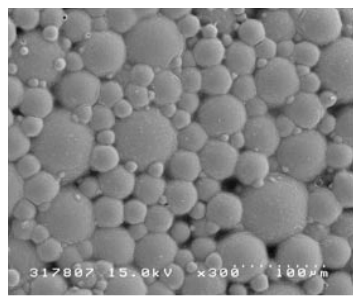

$5 \mathrm{~min}$

Fig. 1 SEM images of the surfaces of Unifast III after curing in a dental pressure pot. Time points at which the specimen was placed in the pressure pot after starting the filling are shown.

points, namely, $5 \mathrm{~mm}, 12.5 \mathrm{~mm}$, and $20 \mathrm{~mm}$ from the left edge. Dimensional accuracy was assessed as the polymerization shrinkage rate $(P S R) \mathrm{cal}^{-}$ culated from the mean value of the gap $(d)$ ac- $^{-}$ cording to the following equation:

$$
P S R=d / 24 \text {. }
$$

\section{Three-point bending test}

Forty-eight rectangular parallelepiped specimens $(2 \times 2 \times 25 \mathrm{~mm})$ were prepared by filling a plastic mold with the autopolymerized resin by the brush-on technique. Each group of specimens was polymerized by the aforementioned curing methods. After polishing with a \#600 SiC abrasive paper, all specimens were immersed in water at $37^{\circ} \mathrm{C}$ for 24 hours. After dimensional measurements of the specimens with a micrometer (Digimatic Micrometer; Mitutoyo, Kanagawa, Japan), the 3-point bending tests were performed using a universal testing machine (1123, Instron, Canton, MA) at a crosshead speed of $1.0 \mathrm{~mm} /$ min. The flexural strength $(\sigma)$ was calculated according to the formula

$$
\sigma=3 P l / 2 b h^{2}
$$

by using the distance between the supports $(l=20$ $\mathrm{mm})$, the maximum load $(P)$ exerted on the specimen, and the breadth $(b)$ and height $(h)$ of the specimen.

\section{Tensile bond test}

Figure 3 shows the schematic illustration of a

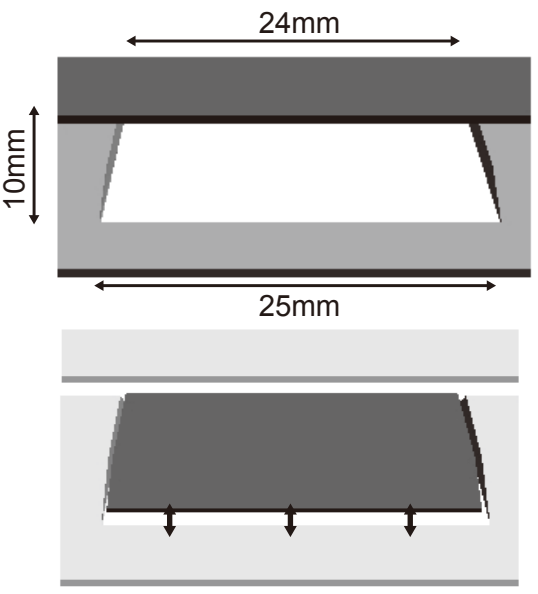

Fig. 2 Schematic illustration of a terinary stainless-steel mold and specimen.
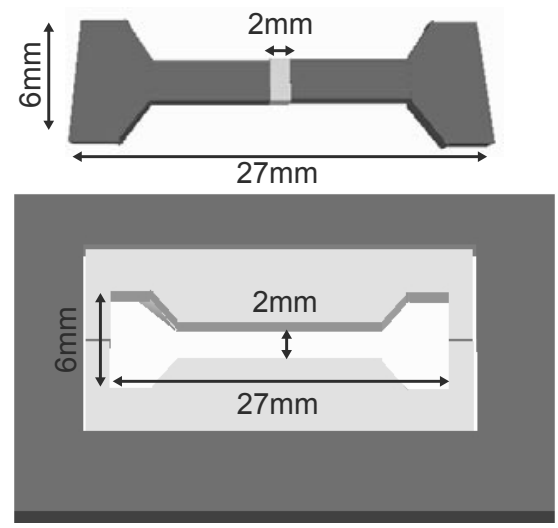

Fig. 3 Schematic illustration of a dumbbell-shaped stainless-steel mold and specimen. 
Table 2 Means and standard deviations of polymerization shrinkage rate (PSR), flexural strength, and tensile bond strength.

\begin{tabular}{|c|c|c|c|c|c|c|c|c|}
\hline & \multicolumn{2}{|c|}{ PSR (\%) } & \multicolumn{2}{|c|}{ Flexural strength $(\mathrm{MPa})$} & \multicolumn{2}{|c|}{ Flexural stlength $(\mathrm{MPa})$} & \multicolumn{2}{|c|}{ Tensile bond strength $(\mathrm{MPa})$} \\
\hline & Non-pressure & Pressure & Non-pressure & Pressure & Non-pressure & Pressure & Non-pressure & Pressure \\
\hline Unifast II & $\begin{array}{c}0.51 \\
(0.10)\end{array}$ & $\begin{array}{c}0.22 \\
(0.06)\end{array}$ & $\begin{array}{c}60.51 \\
(3.66)\end{array}$ & $\begin{array}{c}61.67 \\
(4.55)\end{array}$ & $\begin{array}{c}1799.00 \\
(126.36)\end{array}$ & $\begin{array}{c}1.73 \\
(0.14)\end{array}$ & $\begin{array}{c}9.48 \\
(1.91)\end{array}$ & $\begin{array}{c}8.89 \\
(2.60)\end{array}$ \\
\hline Unifast III & $\begin{array}{c}0.31 \\
(0.06)\end{array}$ & $\begin{array}{c}0.26 \\
(0.05)\end{array}$ & $\begin{array}{c}55.23 \\
(3.55)\end{array}$ & $\begin{array}{l}59.2 \\
(4.03)\end{array}$ & $\begin{array}{c}1614.67 \\
(153.89)\end{array}$ & $\begin{array}{c}1.75 \\
(0.10)\end{array}$ & $\begin{array}{c}9.13 \\
(2.70)\end{array}$ & $\begin{array}{c}8.50 \\
(2.60)\end{array}$ \\
\hline Provinice & $\begin{array}{c}0.35 \\
(0.02)\end{array}$ & $\begin{array}{c}0.23 \\
(0.08)\end{array}$ & $\begin{array}{c}60.50 \\
(5.60)\end{array}$ & $\begin{array}{c}61.89 \\
(3.13)\end{array}$ & $\begin{array}{r}1619.50 \\
(94.53)\end{array}$ & $\begin{array}{c}1.62 \\
(0.11)\end{array}$ & $\begin{array}{c}14.25 \\
(3.17)\end{array}$ & $\begin{array}{c}16.84 \\
(3.93)\end{array}$ \\
\hline Miky Plus & $\begin{array}{c}0.41 \\
(0.04)\end{array}$ & $\begin{array}{c}0.25 \\
(0.06)\end{array}$ & $\begin{array}{c}64.10 \\
(10.35)\end{array}$ & $\begin{array}{l}72.26 \\
(2.33)\end{array}$ & $\begin{array}{c}1613.67 \\
(130.27)\end{array}$ & $\begin{array}{c}1.72 \\
(0.07)\end{array}$ & $\begin{array}{c}14.09 \\
(3.75)\end{array}$ & $\begin{array}{c}13.15 \\
(6.07)\end{array}$ \\
\hline
\end{tabular}

dumbbell-shaped stainless-steel mold and specimen. ${ }^{11}$ The dumbbell-shaped specimen has a parallel section measuring $2.0 \times 2.0 \times 16.0 \mathrm{~mm}$ at the center. Forty-eight dumbbell-shaped specimens were prepared from a conventional heatpolymerizing resin (Acron; GC, Tokyo, Japan) in a constant-temperature water bath at $74^{\circ} \mathrm{C}$ for 8 hours, according to the manufacturer's instructions. All specimens were cut with a low'speed diamond saw (Isomat; Buehler, Lake Bluff, IL) at the center, and the sectioned surfaces were polished with a \#600 SiC abrasive paper. Deposits on the specimens were removed by cleaning in distilled water for 5 minutes. The sectioned dumbbell-shaped specimens were placed into the dumbbell-shaped stainless steel mold. The gap between the 2 pieces of the specimens was approximately $2.0 \mathrm{~mm}$. Autopolymerized resin was filled into the remaining space of the mold by the brush-on technique and polymerized with or without pressure. After immersing in water at $37^{\circ} \mathrm{C}$ for 24 hours, the size of the bonded area was measured with a micrometer. The fracture load of the specimen was determined using a micro-material testing machine (MMT-250N; Shimadzu, Kyoto, Japan) at a crosshead speed of $1.0 \mathrm{~mm} / \mathrm{min}$. The tensile bond strength was calculated as the fracture load divided by the original cross-sectional area.

\section{Statistical analysis}

Statistical analysis was performed using JMP ver. 6.0 (SAS, Cary, NC). Data were statistically analyzed using two-way analysis of variance (ANOVA) in combination with Tukey's multiple comparison test at a significance level of $\alpha=0.05$.

\section{Results}

\section{Polymerization shrinkage rate}

Table 2 shows the means and standard deviations of PSR. Two-way ANOVA indicated that both resin and pressure had significant effects on the mechanical properties of autopolymerized resins (Table 3). The interaction between resin and pressure was also significant. The shrinkage was lower in the pressure group than in the nonpressure group (Fig. 4). Unifast III had the lowest PSR in the non-pressure group, while there were no significant differences in PSR in the pressure group. The PSRs of Unifast II and Miky Plus greatly improved under pressure, while those of Unifast III and Provinice showed slight improvements (Fig. 5).

\section{Flexural strength}

Significant differences in flexural strength were observed between the pressure group and the non-pressure group (Tables 2 and 4). There was no interaction between resin and pressure. The flexural strength increased when the specimens were cured in the pressure pot (Fig. 6).

\section{Tensile bond strength}

After the tensile bond test, the specimens showed interfacial fractures. There were no significant differences in tensile bond strength between the pressure group and the non-pressure group, but there were significant differences among resins (Tables 2 and 5). Provinice and Miky Plus showed greater tensile bond strength than Unifast II and Unifast III (Fig. 7). 
Table 3 Summary of two-way analysis of variance for polymerization shrinkage rate.

\begin{tabular}{cccccc}
\hline Source & df & Sum of squares & Mean square & $F$-value & $P$-value \\
\hline Resin (a) & 3 & 0.049 & 0.016 & 4.157 & 0.012 \\
Pressure (b) & 1 & 0.281 & 0.281 & 71.648 & $<.0001$ \\
a $\times b$ & 3 & 0.089 & 0.030 & 7.555 & $<.0001$ \\
Error & 40 & 0.157 & 0.004 & & \\
\hline
\end{tabular}

Table 4 Summary of two-way analysis of variance for flexural strength.

\begin{tabular}{cccccc}
\hline Source & df & Sum of squares & Mean square & $F$-value & $P$-value \\
\hline Resin (a) & 3 & 750.781 & 250.260 & 9.236 & $<.0001$ \\
Pressure (b) & 1 & 161.443 & 161.443 & 5.958 & 0.019 \\
a×b & 3 & 95.059 & 31.686 & 1.169 & 0.333 \\
Error & 40 & 1083.900 & 27.098 & & \\
\hline
\end{tabular}

Table 5 Summary of two-way analysis of variance for tensile bond strength.

\begin{tabular}{cccccc}
\hline Source & df & Sum of squares & Mean square & $F$-value & $P$-value \\
\hline Resin (a) & 3 & 393.512 & 131.171 & 10.403 & $<.0001$ \\
Pressure (b) & 1 & 0.150 & 0.150 & 0.012 & 0.914 \\
a×b & 3 & 28.426 & 9.475 & 0.752 & 0.528 \\
Error & 40 & 504.377 & 12.609 & & \\
\hline
\end{tabular}
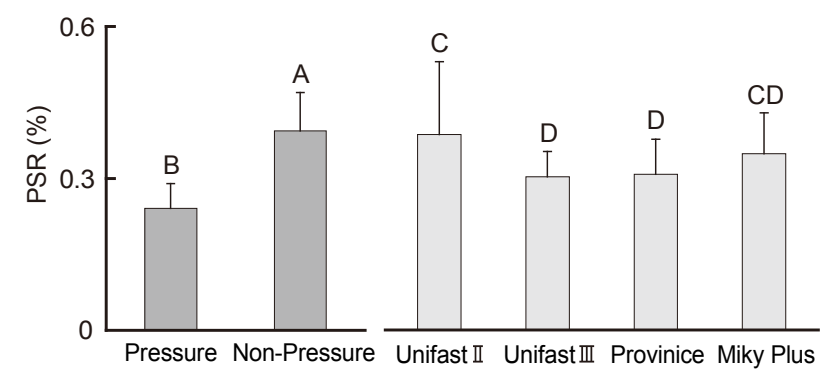

Fig. 4 Effect of resin and pressure on polymerization shrinkage rates (PSRs). Bars with the same superscript are not significantly different $(P>0.05)$. Error bars indicate $95 \%$ confidential intervals.

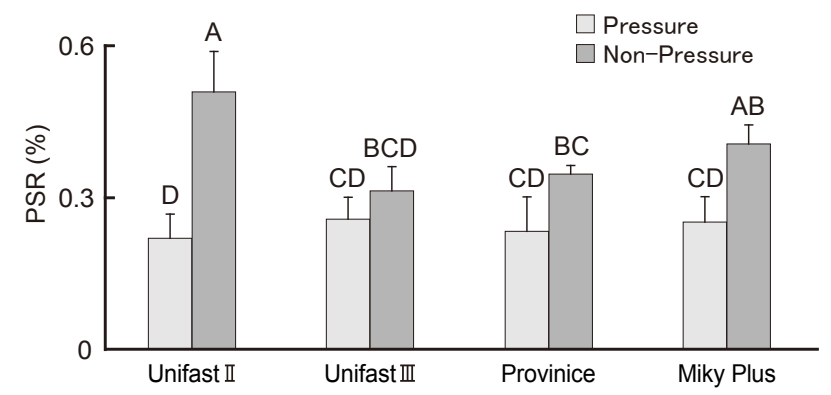

Fig. 5 Effect of the interaction of resin and pressure on polymerization shrinkage rates (PSRs). Bars with the same superscript are not significantly different $(P>0.05)$. Error bars indicate $95 \%$ confidential intervals.

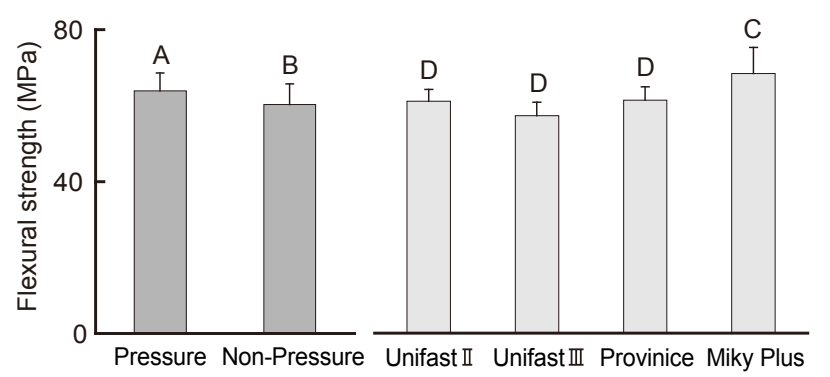

Fig. 6 Effect of resin and pressure on flexural strength. Bars with the same superscript are not significantly different $(P>0.05)$. Error bars indicate $95 \%$ confidential intervals.

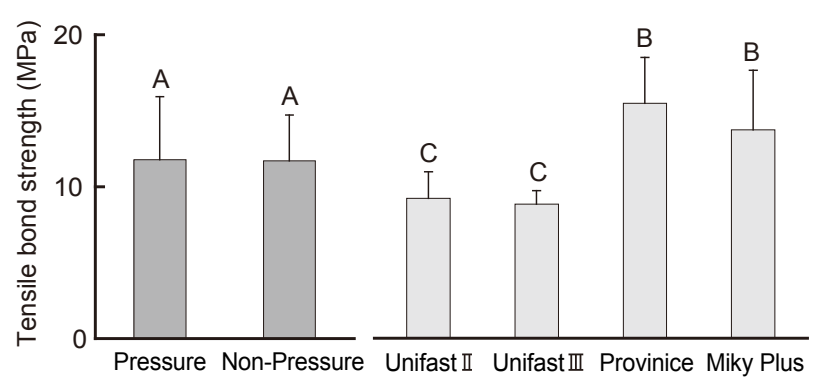

Fig. 7 Effect of resin and pressure on tensile strength. Bars with the same superscript are not significantly different $(P>0.05)$. Error bars indicate $95 \%$ confidential intervals. 


\section{Discussion}

In the preliminary experiment, when the specimens were subjected to pressure-pot polymerization within 1 minutes, their surfaces became smooth. The amount of monomer evaporating from the mixture could be lower in pressure-pot polymerization than in on-bench polymerization. Surface examination of the specimens revealed that polymers and monomers were well mixed and dissolved in pressure-pot polymerization, while only polymers were apparent and were less dissolved in on-bench polymerization.

The PSR improved under pressure curing because the direction of shrinkage was only from the upper surface toward the center. Meanwhile, on-bench polymerization without pressure had disadvantages with respect to dimensional accuracy because polymerization shrinkage occurred from all directions toward the center. The doughing times and curing times of each of the 4 resins were different. ${ }^{12}$ Pressure has the greatest influence on dimensional accuracy at the dough phase. Unifast III had the shortest curing time among the 4 resins. Therefore, pressure had greater influence on resins with longer doughing and curing times.

In the 3-point bending test, the pressure group showed greater flexural strength than the nonpressure group. The increased strength was the result of a decrease in the size and number of internal porosities produced by pressure application during polymerization.

Tensile bond strength remained low irrespective of pressure application, and no significant differences were observed between the pressure group and the non-pressure group. This is because it is difficult for the monomers of the autopolymerized resin to dissolve the polymers of the heat-polymerized resin within the curing time of the autopolymerized resin. ${ }^{13}$ Thus, coating with a primer such as dichloromethane is recommended to obtain greater tensile bond strength while repairing dentures. ${ }^{14,15}$ Provinice and Miky Plus have slightly greater tensile bond strengths than Unifast II and III. The doughing times of Provinice and Miky Plus were longer than those of Unifast II and III. A longer doughing time means that the surface of the denture base resins might be affected to a greater extent by the monomers in the autopolymerized resin, which will contribute to better tensile bond strength.

The results of this study suggest that the di- mensional accuracy and flexural strength of autopolymerized resins are significantly improved by the application of water pressure alone at room temperature. Water pressure curing will contribute to the longevity and better aesthetic appearance of provisional restorations and repaired dentures. However, attention should be paid to the effect of pressure on the type of material used, particularly with respect to its original properties such as doughing time and curing time. The influence of other factors such as air or water pressure, temperature during polymerization, and application of denture primer on the mechanical properties of autopolymerized resins will be investigated in future studies.

\section{Conclusion}

The dimensional accuracy and flexural strength of autopolymerized resins were significantly improved by the application of water pressure during polymerization at room temperature. However, there was no significant difference in tensile bond strength between the pressure and non-pressure groups at room temperature.

\section{References}

1. Donovan TE, Hurst RG. Physical properties of acrylic resin polymerized by four different techniques. J Prosthet Dent 54: 522-524, 1985.

2. Yunus N, Harrisons A, Huggett R. Effect of microwave irradiation on the flexural strength and residual monomer levels of an acrylic resin repair material. J Oral Rehabil 21: 641-648, 1994.

3. Ogawa T, Hasegawa A. Effect of curing environment on mechanical properties and polymerizing behavior of methyl-methacrylate autopolymerizing resin. J Oral Rehabil 32: 221-226, 2005.

4. Renner RP, Boucher LJ. Removable Partial Dentures 359-389, Chicago: Quintessence, 1987.

5. Zarb GA, Bolender CL et al. Boucher's prosthodontic treatment for edentulous patients $10^{\text {th }} \mathrm{ed}$. 587-594, St. Louis: CV. Mosby, 1990.

6. Ware AL, Docking AR. The strength of acrylic repairs. Aust Dent J 54: 27-32, 1950.

7. Sanford JW, Burns CL, Paffenbarger GC. Selfcuring resins for repairing dentures: some physical properties. J Am Dent Assoc 51: 301-315, 1955.

8. McCrorle JW, Anderson JN. Transverse strength repairs with self-curing resins. Br Dent J 109: 364-366, 1960.

9. Beyli MS, Von Fraunhofer JA. Repair of fractured 
acrylic resin. J Prosthet Dent 44: 497-503, 1980.

10. Word JE, Moon PC, Behrendt CL. Effect of repair surface design, repair material, and processing method on the transverse strength of repaired acrylic denture resin. J Prosthet Dent 67: 815-820, 1992.

11. Sato T, Takahahi H, Hongo T et al. Effect of degradation of denture base resin on bond strength to relining resins. Dent Mater J 26: 89-95, 2007

12. Vallittu PK, Ruyter IE. Swelling of poly(methyl methacrylate) resin at the repair joint. Int $\mathrm{J}$ Prosthodont 10: 254-258, 1997.

13. Kamada N, Ohkubo C, Shimpo H et al. Fluidity and hardening time of autopolymerized denture repair acrylic resin. A comparison of brush-on technique and mixing technique. Prosthodont Res Pract, in press.

14. Arima T, Nikawa H, Hamada T et al. Composition and effect of denture base resin surface primers for reline acrylic resins. J Prosthet Dent 75: 457-462, 1996.

15. Minami H, Suzuki S, Minesaki Y et al. In vitro evaluation of the influence of repairing condition of denture base resin on the bonding of autopolymerizing resins. J Prosthet Dent 91: 164-170, 2004. 